\title{
Evaluación del uso de las TIC en estudiantes de la Universidad de Málaga: diferencias de género
}

\section{Evaluation in the use of TIC in students of the University of Malaga: gender differences}

\author{
Lourdes Aranda ${ }^{1}$, Laura Rubio ${ }^{2}$, Cristina Di Giusto Valle ${ }^{3}$, Cristina Dumitrache ${ }^{4}$ \\ ${ }^{1}$ Departamento de THE y Métodos de Investigación y Diagnóstico en Educación, Universidad de Málaga, España \\ (maragar@uma.es) \\ ${ }^{2}$ Departamento de Psicología Evolutiva y de la Educación, Universidad de Granada, España (lrrubio@ugr.es) \\ ${ }^{3}$ Departamento de Educación, Universidad de Burgos, España (cdi@ubu.es) \\ ${ }^{4}$ Departamento de Psicología Evolutiva y de la Educación, Universidad de Granada, España (cgdumitrache@ugr.es)
}

Recibido el 2 de agosto de 2018; revisado el 28 de septiembre de 2018; aceptado el 1 de octubre de 2018 ; publicado el 1 de junio de 2019

\section{RESUMEN:}

Con la implantación del Espacio Europeo de Educación Superior (EEES), una de las cuestiones más relevantes que se incluyen en la formación universitaria es el manejo de las nuevas tecnologías de la información y la comunicación (TIC) por parte del alumnado. En esta investigación se ha evaluado el uso de las TIC en 79 estudiantes $\left(M_{\text {edad }}=20,43\right.$, $D T=3,83)$ de primer curso de Pedagogía de la Universidad de Málaga, y se han analizado las diferencias de género en el ámbito tecnológico. Para la recogida de datos se usó un cuestionario tipo Likert que recababa información sobre las características sociodemográficas y sobre el conocimiento y uso de las TIC. Los resultados muestran una clara diferencia de género en el conocimiento y manejo de las herramientas tecnológicas más complejas, siendo las mujeres las que tienen un conocimiento más escaso. Por otra parte, los resultados muestran que los estudiantes que usan las aulas informáticas universitarias presentan mejores habilidades en el funcionamiento de los foros universitarios que los estudiantes que no usan dichas aulas, independientemente de que sean hombres o mujeres.

\section{PALABRAS CLAVE: ENSEÑANZA SUPERIOR, TIC, PROCESO DE APRENDIZAJE, INVESTIGACIÓN CUANTITATIVA.}

\begin{abstract}
:
One of the most important changes in the University Education System after the implementation of the European Higher Education Area (EHEA), is the management of new Information and Communication Technologies (ICT) by students. In this research the use of ICT was evaluated in 79 students $\left(M_{\text {age }}=20,43, S D=3,83\right)$ of the University of Malaga who were studying the first course of the Pedagogy Degree. Gender differences in the technological field were analyzed. For the collection of data, a Likert questionnaire was used that gathered information about sociodemographic characteristics and knowledge and use of new ICT. Results show a clear gender difference in knowledge and management of the most complex technological tools, with women having the most limited knowledge. On the other hand, the results show that students who use the University's computer rooms present better skills in the functioning of University forums than students who do not use such classrooms, regardless of whether they are men or women.
\end{abstract}


KEYWORDS: HIGHER EDUCATION, ICT, LEARNING PROCESSES, QUANTITATIVE RESEARCH

\section{INTRODUCCIÓN}

La comunidad universitaria europea ha experimentado grandes cambios debido a la implantación del Espacio Europeo de Educación Superior (EEES). Por ello, las instituciones educativas, profesores y estudiantes, han tenido que modificar su forma de trabajar, cambiando así el papel que desempeñan en los procesos de enseñanza-aprendizaje en las diferentes titulaciones de la universidad.

En este nuevo marco Europeo de la Educación Superior hemos de destacar varios cambios en los procesos de aprendizaje en todas las titulaciones universitarias de los estados europeos: uno de los cambios relevantes es el referente a la metodología y diseño curricular utilizados por los docentes (Pozo y Bretones, 2015; Rodríguez, Álvarez, Gil y Romero, 2011), que deben impartir clases enfocadas a la resolución de problemas reales en lugar de clases magistrales donde el alumno se limita a escuchar al docente sin apenas participar, tomando así un papel pasivo. Este aspecto es de especial importancia en la convergencia al EEES, ya que los alumnos pasan a ser autónomos y participativos y, en definitiva, agentes activos en todo su proceso formativo, en contraposición al papel que tenían en la enseñanza superior tradicional (Aranda et al., 2015; Santacruz, Aranda-Garrido y García-Moreno, 2013).

Por otra parte, un aspecto importante a destacar en el EEES es la introducción del sistema ECTS (European Credit Transfer System) como un sistema de transferencia y acumulación de créditos. El ECTS es un sistema de créditos que, por un lado, considera a los alumnos el eje principal de los procesos de enseñanza-aprendizaje $\mathrm{y}$, por otro, valora el número de horas trabajadas por estos. Esta acumulación de horas es imprescindible para la consecución de los objetivos necesarios de programas específicos, dotando así a los estudiantes de las competencias para completar su formación universitaria (Comas, 2013; Gijón y Crisol, 2012). Como consecuencia de ello, el diseño curricular en la enseñanza superior ya no se basa en los contenidos, sino que está basado en la adquisición de competencias, donde el alumno debe obtener unos niveles mínimos de destrezas y habilidades para, más tarde, poder aplicarlo en contextos sociales o laborales (Santacruz et al., 2015; Vidal, 2012). En este sentido, las políticas educativas deben responder a las necesidades laborales y, en general, a las necesidades sociales que van cambiando continuamente $y$, de esta forma, preparar al alumnado para cubrir tales necesidades.

Actualmente, son muchos los autores que definen "la competencia" dentro del espacio europeo universitario. En este contexto, hemos querido resaltar la definición dada por Tobón, Pimienta y García (2010) que la consideran como: "actuaciones integrales para identificar, interpretar, argumentar y resolver problemas con idoneidad y compromiso ético, movilizando los diferentes saberes: ser, hacer y conocer" (p. 11).

Con estos cambios todos los países europeos unifican sus procedimientos de aprendizaje y, por tanto, se está experimentando una transformación en la metodología didáctica de la enseñanza superior. Los docentes y estudiantes comienzan a usar las tecnologías de la información y la comunicación (TIC), que les son de gran utilidad en los procesos de enseñanza-aprendizaje (Fernández-Cruz, Fernández-Díaz y Rodríguez-Mantilla, 2018; Morales, Ortíz, Trujillo y Raso, 2015). De hecho, la integración y uso de las TIC en las universidades españolas ha supuesto una mejora importante en los procesos de enseñanza-aprendizaje (HernándezSellés, 2015).

Grande, Cañón y Cantón (2016) destacan las diferentes definiciones que nos encontramos en la literatura sobre las TIC: "componente relativo al desarrollo tecnológico (los dispositivos, la industria, etc.) y sus consecuencias para la gestión de la información, nuestra manera de interiorizar el entorno, de abordar la realidad y comunicarnos" (p. 6).

Investigaciones previas, muestran que las TIC son herramientas de gran utilidad y relevancia en la comunidad universitaria, ya que facilitan el aprendizaje significativo además de fomentar la investigación y formar a futuros profesionales competentes, adaptados a la sociedad actual (Morales et al., 2015; Gudín de la Lama, LasalaNavarro y Iturriaga-Barco, 2016). Sin embargo, el uso de las TIC no significa que aporte nuevos conocimientos o principios educativos en los diferentes grados incluidos en enseñanza superior, sino que facilita de forma notable el proceso educativo.

Aunque la incorporación de las herramientas TIC facilita de forma significativa la adquisición de competencias al alumnado, no es menos cierto que su inclusión en los procesos de enseñanzaaprendizaje requiere de importantes cambios en la comunidad universitaria (Díaz-Levicoy, 2013; López-Navas, 2014; Nakano, Garret, Vásquez y 
Mija, 2014). Pues bien, son varias las dificultades que se presentan para la inclusión de estas herramientas, donde podemos destacar la disponibilidad tecnológica y la formación adecuada en TIC por parte de docentes y de estudiantes. Centrándonos en la última dificultad, son muchos los estudios que señalan la necesidad de la formación en TIC de los docentes, ya que no todos los docentes poseen una formación efectiva, eficiente y adecuada (Honmy y Vázquez, 2012) y, por tanto, presentan unos índices altos de desmotivación. Aun así, tanto docentes como alumnado consideran que el uso de las herramientas TIC es fundamental en la educación superior (Giudicessi, Martínez-Ceron, Saavedra, Cascone, Camperi, 2016).

Por lo tanto, el uso de estas herramientas de enseñanza-aprendizaje introduce importantes mejoras en la calidad formativa de los estudiantes de nuestras Universidades (García-Sánchez, ReyesAñorve y Godínez-Alarcón, 2017), haciéndose cada vez más necesaria su aplicación en los diferentes centros de enseñanza superior.

Por ello, el objetivo de este estudio es medir el conocimiento y uso de las TIC en estudiantes de primer curso de Pedagogía de la Universidad de Málaga, así como estudiar las posibles diferencias en el ámbito tecnológico entre los alumnos y alumnas de dicho Grado.

\section{MATERIAL Y MÉTODO}

La metodología empleada en esta investigación ha sido cuantitativa-descriptiva, donde se realizó un diseño de investigación de tipo transversal.

\section{1 Participantes}

La muestra de este estudio estuvo compuesta por 79 estudiantes, de los cuales 14 eran hombres $(17,7 \%$ de la muestra) y 65 mujeres (82,3\% de la muestra). La edad de los estudiantes oscilaba entre 18 y 36 años $\left(M_{\text {edad }}=20,43, D T=3,83\right)$.

Los participantes de esta investigación pertenecían al primer curso del Grado en Pedagogía de la Facultad de Ciencias de la Educación de la Universidad de Málaga durante el curso 2017/2018.

El tipo de muestreo utilizado ha sido no probabilístico de tipo incidental, para poder acceder fácilmente a la muestra seleccionada.

\subsection{Instrumento de recogida de información}

La recogida de información se realizó a través de un instrumento de encuesta, concretamente se utilizó una adaptación del Cuestionario de Competencias Tecnológicas de los alumnos/as (Llorente-Cejudo y Cabero, 2010). Es un instrumento de tipo Likert de 71 ítems con una escala de respuesta de 1 a 5 , donde el 1 representa que el alumno/a se siente completamente ineficaz para realizar lo que presenta el ítem y 5 que cree que lo domina completamente.

En este cuestionario los 8 primeros ítems son variables sociodemográficas y los ítems 9-71 corresponden a cuestiones sobre percepciones y uso de las TIC. Las dimensiones en las que se dividieron los ítems de desarrollo de este instrumento de recogida de datos fueron los siguientes (adaptado de Llorente-Cejudo y Cabero, 2010):

Dimensión 1: conocimiento básico en el manejo de herramientas tecnológicas. En esta primera dimensión se incluyeron los ítems:

- I-9. Tengo conocimientos básicos sobre el funcionamiento de un ordenador y sus periféricos.

- I-10. Sé conectar un ordenador y sus periféricos más usuales: impresoras, escáner, etc.

- I-11. Sé conectar equipos de audio, cámaras de vídeo y fotos digitales a los ordenadores.

- I-16. Realizo un documento escrito con un procesador de texto (Word, WordPerfect, Writer, Abiword, etc.).

- I-17. Realizo un documento escrito con un procesador de texto (Word, WordPerfect, Writer, Abiword, etc.), usando técnicas avanzadas del mismo para: poner encabezamiento, cambiar el tipo y tamaño de letra, poner negrillas, subrayados, etc.

- I-18. Sé realizar un documento escrito con un procesador de texto (Word, WordPerfect, Writer, Abiword, etc.), usando sus posibilidades de insertar tablas, gráficos o textos de otros documentos.

- I-30. Identifico aspectos de estilos en una presentación (PowerPoint, Impress, etc.) realizada por otra persona.

- I-32. Navego por Internet con diferentes navegadores: Chrome, Firefox, Opera, etc.

- I-33: Navego por Internet mediante los distintos links, enlaces o hipervínculos que proporcionan las páginas webs que voy visitando.

- I-36. Soy capaz de descargar de Internet, programas, imágenes, clips de audio, etc.

- I-39. Soy capaz de utilizar diferentes buscadores de Internet (Google, Yahoo, etc.).

- I-41. Puedo organizar la información recogida de Internet, agregando las páginas que me interesan a favoritos $y$ clasificarlas en subcarpetas bajo algún criterio de ordenación. 
- I-45. Me puedo comunicar con otras personas, por correo electrónico, chat, mensajería instantánea, listas de distribución, etc. es decir, mediante las herramientas de comunicación usuales de Internet;

- I-62. Sé utilizar los correctores ortográficos de los procesadores de texto, para editar y revisar mis trabajos.

- I-65. Sé crear una cuenta de correo electrónico a través de diferentes programas: Yahoo, Hotmail, Gmail, etc.

Dimensión 2. Conocimiento medio en el manejo de las herramientas tecnológicas. En la segunda dimensión se incluyen los ítems:

- I-13. Sé usar de forma apropiada combinaciones de teclas para conseguir signos alfanuméricos y de puntuación desde el teclado.

- I-14. Soy capaz de instalar y desinstalar programas informáticos en un ordenador.

- I-15. Puedo cambiar de formatos los ficheros (convertir un fichero de un tipo a otro).

- I-19. Sé realizar consultas sobre bases de datos elaboradas por otros.

- I-23. Sé diseñar, crear y modificar hojas de cálculo con algún programa informático (Excel, Calc, Gnumeric, etc.), para propósitos específicos, usando sus funciones como dar formato a las celdas, insertar y ocultar filas, realizar tablas dinámicas.

- I-29. Sé crear una presentación multimedia mediante algún programa, incluyendo imágenes estáticas, textos, clip de audio, clip de vídeo, gráficas, etc.

- I-38. Soy capaz de coordinar una actividad en grupo realizada en Internet, por ejemplo un foro electrónico.

- I-40: Soy capaz de utilizar las opciones de búsqueda avanzada ("y" - "o") en diferentes buscadores de Internet (Google, Yahoo,...) para refinar la búsqueda de información;

- I-43: Soy capaz de realizar videoconferencias por IP (Netmeeting, Messenger,...) a través de Internet;

- I-44: Soy capaz de acceder, buscar y recuperar información utilizando diferentes formas de accesibilidad y formatos (CD-ROM, DVD, vídeo,...);

- I-46: Soy capaz de organizar, analizar y sintetizar la información mediante tablas, gráficos o esquemas;

- I-48: Sé usar organizadores gráficos, tales como mapas de pensamiento, diagramas o esquemas, para presentar las relaciones entre ideas o conceptos;

- I-50: Conozco programas informáticos para compartir información en la red con mis compañeros;

- I-51: Conozco las herramientas que me proporciona el sistema operativo para compartir recursos en la red del aula (carpetas, unidades, periféricos,...);

- I-53. Soy capaz de usar las TIC para investigar, explorar, interpretar información o resolver problemas en diversidad de materias y contextos.

- I-54. Soy capaz de evaluar la autoría y fiabilidad de la información encontrada en Internet; es decir, evaluar la relevancia de la información localizada en Internet.

- I-55. Sé explicar las ventajas y limitaciones que presentan los ordenadores para almacenar, organizar recuperar y seleccionar información.

- I-58. Me considero competente para saber discriminar en la mayoría de los casos, correo electrónico con virus, basura o spam.

- I-59. Me siento capaz de evaluar la efectividad de los usos que yo y mis compañeros hacemos de las fuentes de información y de las herramientas de las TIC, para mejorar la calidad de los trabajos de clase.

- I-60. Soy capaz de realizar búsquedas bibliográficas a través de diferentes bases de datos disponibles en la red.

- I-61. Sé utilizar herramientas y recursos de la tecnología para administrar y comunicar información personal y/o profesional.

Dimensión 3: Conocimiento avanzado en el manejo de las herramientas tecnológicas. En esta dimensión los ítems son:

- I-12. Resuelvo problemas como configurar el correo electrónico, configurar antivirus, desfragmentar el disco duro, etc., que se presenten en el ordenador o en Internet.

- I-20. Sé diseñar, crear y modificar bases de datos con algún programa informático (Access, Base, Knoda, MySQL, etc.), para propósitos específicos.

- I-21. Sé diseñar, crear y modificar bases de datos con algún programa informático (Access, Base, Knoda, MySQL, etc.), para propósitos específicos donde se utilicen formularios, informes asociados a una tabla, se creen macros asociados a los controles del formulario, es decir, de forma avanzada. 
- I-22. Sé diseñar, crear y modificar hojas de cálculo con algún programa informático (Excel, Calc, Gnumeric, etc.), para propósitos específicos, usando sus funciones elementales como las de suma, productos, o medias.

- I-24. Diseñar, crear y modificar hojas de cálculo con algún programa informático (Excel, Calc, Gnumeric, etc.), para propósitos específicos, usando fórmulas o funciones.

- I-25. Sé usar diferentes programas que incluyen herramientas para dibujar gráficos.

- I-26. Uso las calculadoras científicas que incluyen los sistemas operativos para resolver problemas numéricos.

- I-27. Sé crear imágenes y gráficos mediante algún programa informático.

- I-28. Sé crear clip de audio con algún programa informático.

- I-31. Sé modificar imágenes mediante algún programa de diseño gráfico (CorelDRAW, Photoshop, GIMP, etc.).

- I-34. Sé diseñar páginas web, utilizando algún programa informático, incluyendo textos, imágenes, etc.

- I-35. Sé diseñar páginas web utilizando algún programa informático, incluyendo diferentes links, al propio documento o a otros.

- I-37. Sé usar software de trabajo colaborativo.

- I-42. Sé enviar ficheros de un ordenador a otro por Internet mediante FTP.

- I-47. Soy capaz de organizar la información, usando herramientas como bases de datos, hojas de cálculo o programas similares.

- I-49. Sé utilizar manuales de ayuda en línea.

- I-52. Me siento competente para reconocer dónde es conveniente elaborar grupos de instrucciones y a automatizar procesos de uso frecuente mediante macros, procedimientos de control, uso de fórmulas, etc.

- I-56. Comprendo los problemas de compatibilidad entre hardware y software informático.

- I-57. Me considero competente para saber juzgar y hacer aportaciones para mejorar las producciones multimedia, realizadas por mis compañeros.

Dimensión 4. Uso y conocimientos de los Laboratorios Tecnológicos y Enseñanza Virtual de la Universidad de Málaga. Corresponden a esta dimensión los ítems:

- I-63. Sé utilizar la plataforma de enseñanza virtual de la Universidad de Málaga.
- I-64. Me encuentro competente al utilizar el servicio de consignas para bajar ficheros de la Universidad de Málaga.

- I-66. Sé crear mi cuenta de correo en el dominio de la Universidad de Málaga (uma.es).

- I-67. Sé acceder a mi expediente académico virtual en la Universidad de Málaga.

- I-68. Sé utilizar los foros de la comunidad universitaria o de los alumnos de la Universidad de Málaga.

- I-69. Sé registrar documentación a través del registro telemático de la Universidad de Málaga.

- I-70. Me considero capaz de localizar la dirección de correo electrónico y los teléfonos de los profesores de la Facultad de Pedagogía a través del directorio de su página web.

- I-71. Sé utilizar los diferentes recursos electrónicos (catálogo, revistas electrónicas, bases de datos, etc.) disponibles en la biblioteca virtual de la Universidad de Málaga.

Los participantes respondieron a 8 preguntas sociodemográficas tales como la edad y el género, además de otras cuestiones de interés sobre la posesión y manejo de herramientas tecnológicas (ver tabla 1).

Tabla 1. Uso de los dispositivos tecnológicos de los que dispone el alumnado.

Frecuencia \%

¿Tienes ordenador de mesa en casa?

Sí..................................

No............................

$2 \quad 2,5$

¿Tienes conexión a Internet en casa?

Sí.

$79 \quad 100$

No...

0

0

¿Posees un portátil?

Sí.

$70 \quad 100$

No.

0

0

Ordenador en que realizas los trabajos

En el mío...

En el ordenador de la Facultad......

Otros.

79

100

$0 \quad 0$

$0 \quad 0$

¿Utilizas el aula de informática de la Facultad?

Sí....

No. 


\section{RESULTADOS}

Todos los análisis estadísticos se realizaron usando el programa para tratamiento de datos IBM SPSS V. 22.

Las variables dependientes registradas fueron los "ítems del cuestionario" referidos al conocimiento y manejo de las TIC, mientras que las variables independientes fueron género y uso del aula de informática.

Se realizó un análisis descriptivo donde se obtuvieron las frecuencias y porcentajes de las diferentes respuestas de los ítems que componían el cuestionario. Se agruparon las opciones de respuesta 1 (completamente ineficaz) y 2 (ineficaz), así como la 4 (4ficaz) y 5 (completamente eficaz).

La tabla 2 muestra las frecuencias de respuesta para la dimensión de Conocimiento básico en el manejo de herramientas tecnológicas. En la tabla 3 se encuentran los porcentajes de respuesta para la dimensión de Conocimiento medio en el manejo de las herramientas tecnológicas. La tabla 4 contiene los resultados referentes a la dimensión de Conocimiento avanzado en el manejo de las herramientas tecnológicas. Por último, la tabla 5 resume las frecuencias de respuesta en relación a la dimensión de Uso y conocimientos de los Laboratorios Tecnológicos y Enseñanza Virtual de la Universidad de Málaga.

Tabla 2. Dimensión 1. Conocimiento básico en el manejo de herramientas tecnológicas

\begin{tabular}{lccc}
\hline & $\begin{array}{c}\text { Completamente } \\
\text { ineficaz/Ineficaz } \\
\%\end{array}$ & $\begin{array}{c}\text { Medio } \\
\text { Eficaz } \\
\%\end{array}$ & $\begin{array}{c}\text { Completamente } \\
\text { eficaz/Eficaz } \\
\%\end{array}$ \\
\hline I-9 & $6,3 \%$ & $20,3 \%$ & $73,4 \%$ \\
I-10 & $12,7 \%$ & $25,3 \%$ & $62,0 \%$ \\
I-11 & $6,3 \%$ & $13,9 \%$ & $79,8 \%$ \\
I-16 & $1,3 \%$ & $2,5 \%$ & $96,2 \%$ \\
I-17 & $1,3 \%$ & $0,0 \%$ & $98,7 \%$ \\
I-18 & $0,0 \%$ & $8,8 \%$ & $91,2 \%$ \\
I-30 & $11,4 \%$ & $13,9 \%$ & $74,7 \%$ \\
I-32 & $16,4 \%$ & $8,9 \%$ & $74,7 \%$ \\
I-33 & $6,3 \%$ & $11,4 \%$ & $82,3 \%$ \\
I-36 & $2,5 \%$ & $13,9 \%$ & $83,6 \%$ \\
I-39 & $2,5 \%$ & $3,8 \%$ & $93,7 \%$ \\
I-41 & $13,9 \%$ & $10,1 \%$ & $75,0 \%$ \\
I-45 & $1,3 \%$ & $2,5 \%$ & $96,2 \%$ \\
I-62 & $11,4 \%$ & $17,7 \%$ & $70,9 \%$ \\
I-65 & $6,3 \%$ & $1,3 \%$ & $92,4 \%$ \\
\hline
\end{tabular}

Tabla 3. Dimensión 2. Conocimiento medio en el manejo de las herramientas tecnológicas

\begin{tabular}{cccc}
\hline & $\begin{array}{c}\text { Completamente } \\
\text { ineficaz/Ineficaz } \\
\%\end{array}$ & $\begin{array}{c}\text { Medio } \\
\text { Eficaz } \\
\%\end{array}$ & $\begin{array}{c}\text { Completamente } \\
\text { eficaz/Eficaz } \\
\%\end{array}$ \\
\hline I-13 & $21,5 \%$ & $25,3 \%$ & $53,2 \%$ \\
I-14 & $16,5 \%$ & $21,5 \%$ & $62,0 \%$ \\
I-15 & $15,2 \%$ & $19,0 \%$ & $65,8 \%$ \\
I-19 & $24,0 \%$ & $32,9 \%$ & $43,1 \%$ \\
I-23 & $40,5 \%$ & $26,6 \%$ & $32,9 \%$ \\
I-29 & $15,2 \%$ & $22,8 \%$ & $62,0 \%$ \\
I-38 & $35,4 \%$ & $19,0 \%$ & $45,6 \%$ \\
I-40 & $22,8 \%$ & $19,0 \%$ & $58,2 \%$ \\
I-43 & $31,6 \%$ & $22,9 \%$ & $45,5 \%$ \\
I-44 & $38,0 \%$ & $26,5 \%$ & $35,5 \%$ \\
I-46 & $24,0 \%$ & $22,8 \%$ & $53,2 \%$ \\
I-48 & $39,2 \%$ & $29,1 \%$ & $31,7 \%$ \\
I-50 & $13,9 \%$ & $25,3 \%$ & $60,8 \%$ \\
I-51 & $36,7 \%$ & $19,0 \%$ & $44,3 \%$ \\
I-53 & $13,9 \%$ & $25,3 \%$ & $60,8 \%$ \\
I-54 & $15,2 \%$ & $30,3 \%$ & $54,5 \%$ \\
I-55 & $38,0 \%$ & $27,8 \%$ & $34,2 \%$ \\
I-58 & $24,0 \%$ & $34,2 \%$ & $41,8 \%$ \\
I-59 & $29,1 \%$ & $26,6 \%$ & $44,3 \%$ \\
I-60 & $10,1 \%$ & $35,4 \%$ & $54,5 \%$ \\
I-61 & $32,9 \%$ & $27,3 \%$ & $39,2 \%$ \\
\hline
\end{tabular}

Tabla 4. Dimensión 3. Conocimiento avanzado en el manejo de las herramientas tecnológicas

\begin{tabular}{cccc}
\hline & $\begin{array}{c}\text { Completamente } \\
\text { ineficaz/Ineficaz } \\
\%\end{array}$ & $\begin{array}{c}\text { Medio } \\
\text { Eficaz } \\
\%\end{array}$ & $\begin{array}{c}\text { Completamente } \\
\text { eficaz/Eficaz } \\
\%\end{array}$ \\
\hline $\mathrm{I}-12$ & $35,4 \%$ & $32,9 \%$ & $31,7 \%$ \\
$\mathrm{I}-20$ & $82,3 \%$ & $11,4 \%$ & $6,3 \%$ \\
$\mathrm{I}-21$ & $83,5 \%$ & $11,5 \%$ & $5,0 \%$ \\
$\mathrm{I}-22$ & $39,2 \%$ & $25,3 \%$ & $35,5 \%$ \\
$\mathrm{I}-24$ & $59,5 \%$ & $17,7 \%$ & $22,8 \%$ \\
$\mathrm{I}-25$ & $54,4 \%$ & $2,5 \%$ & $23,1 \%$ \\
$\mathrm{I}-26$ & $57,0 \%$ & $16,4 \%$ & $26,6 \%$ \\
$\mathrm{I}-27$ & $53,2 \%$ & $17,6 \%$ & $29,2 \%$ \\
$\mathrm{I}-28$ & $46,8 \%$ & $25,3 \%$ & $27,9 \%$ \\
$\mathrm{I}-31$ & $38,0 \%$ & $34,1 \%$ & $27,9 \%$ \\
$\mathrm{I}-34$ & $64,6 \%$ & $15,2 \%$ & $20,2 \%$ \\
$\mathrm{I}-35$ & $69,6 \%$ & $15,2 \%$ & $15,2 \%$ \\
$\mathrm{I}-37$ & $54,4 \%$ & $22,8 \%$ & $22,8 \%$ \\
$\mathrm{I}-42$ & $82,3 \%$ & $10,1 \%$ & $7,6 \%$ \\
$\mathrm{I}-47$ & $45,6 \%$ & $17,7 \%$ & $36,7 \%$ \\
$\mathrm{I}-49$ & $57,0 \%$ & $20,2 \%$ & $22,8 \%$ \\
$\mathrm{I}-52$ & $70,5 \%$ & $20,7 \%$ & $8,8 \%$ \\
$\mathrm{I}-56$ & $63,3 \%$ & $21,5 \%$ & $15,2 \%$ \\
$\mathrm{I}-57$ & $44,3 \%$ & $27,8 \%$ & $27,9 \%$ \\
\hline
\end{tabular}


Tabla 5. Dimensión 4. Uso y conocimientos de los Laboratorios Tecnológicos y Enseñanza Virtual de la Universidad de Málaga

\begin{tabular}{cccc}
\hline & $\begin{array}{c}\text { Completamente } \\
\text { ineficaz/Ineficaz } \\
\%\end{array}$ & $\begin{array}{c}\text { Medio } \\
\text { Eficaz } \\
\%\end{array}$ & $\begin{array}{c}\text { Completamente } \\
\text { eficaz/Eficaz } \\
\%\end{array}$ \\
\hline I-63 & $2,5 \%$ & $10,1 \%$ & $87,4 \%$ \\
I-64 & $29,1 \%$ & $30,4 \%$ & $40,5 \%$ \\
I-66 & $3,8 \%$ & $3,8 \%$ & $92,4 \%$ \\
I-67 & $2,5 \%$ & $5,1 \%$ & $92,4 \%$ \\
I-68 & $12,7 \%$ & $16,5 \%$ & $70,8 \%$ \\
I-69 & $44,3 \%$ & $17,7 \%$ & $38,0 \%$ \\
I-70 & $7,6 \%$ & $10,1 \%$ & $82,3 \%$ \\
I-71 & $6,4 \%$ & $27,8 \%$ & $65,8 \%$ \\
\hline
\end{tabular}

Posteriormente, se llevó a cabo un análisis estadístico de los datos mediante la aplicación de la U-Mann-Whitney para muestras independientes en el estudio de la variable ítems del cuestionario, ya que no se cumplió el supuesto básico de homoscedasticidad, observándose distribuciones diferentes entre varianzas.

Los análisis mostraron diferencias estadísticamente significativas $(\mathrm{p}<0,05)$ en los siguientes ítems del cuestionario en función del género:

- Resuelvo problemas como configurar el correo electrónico, configurar antivirus, desfragmentar el disco duro, etc. que se presenten en el ordenador $o$ en Internet $(Z=-2,210 ; p<0,05)$.

- Sé diseñar, crear y modificar bases de datos con algún programa informático (Access, Base, Knoda, MySQL, etc.), para propósitos específicos $(Z=-2,092 ; p<0,05)$.

- Sé diseñar, crear y modificar bases de datos con algún programa informático (Access, Dase, Knoda, MySQL, etc.), para propósitos específicos donde se utilicen formularios, informes asociados a una tabla, se creen macros asociados a los controles del formulario, es decir, de forma avanzada $(Z=-2,109 ; p<0,05)$.

- Sé diseñar, crear y modificar hojas de cálculo con algún programa informático (Excel, Calc, Gnumeric, etc.), para propósitos específicos, usando sus funciones elementales como las de suma, productos, o medias $(\mathrm{Z}=-1,977 ; \mathrm{p}<0,05)$.

- Sé usar software de trabajo colaborativo $(Z=-$ 1,978; $\mathrm{p}<0,05)$.

- Sé utilizar manuales de ayuda en línea $(Z=-2,714$; $\mathrm{p}<0,05)$.

- Comprendo los problemas de compatibilidad entre hardware y software informático ( $\mathrm{Z}=-$ $2,888 ; \mathrm{p}<0,05)$.

- Me considero competente para saber juzgar y hacer aportaciones para mejorar las producciones multimedia, realizadas por mis compañeros $(\mathrm{Z}=-$ $1,978 ; \mathrm{p}<0,05)$.

En todas estas variables dependientes los hombres obtenían unas puntuaciones medias mayores en comparación con las mujeres. Con respecto al resto de ítems no se observaron diferencias estadísticamente significativas entre hombres y mujeres ( $p>0,05)$.

En el caso del análisis estadístico realizado según el Uso del aula universitaria de informática, el análisis mostró diferencias estadísticamente significativas en el ítem "Sé utilizar los foros de la comunidad universitaria o de los alumnos de la Universidad de Málaga”, observándose una media significativamente mayor en el conocimiento de los foros universitarios en aquellos alumnos que habitualmente usaban las aulas universitarias de informática $(Z=-2,495 ; p<0,05)$.

\section{DISCUSIÓN}

Los hallazgos de este estudio muestran las diferencias existentes entre hombres y mujeres en el conocimiento y manejo de las TIC cuando comienzan su formación universitaria. Los datos observados apuntan a que las alumnas poseen un menor dominio que los alumnos en la programación de ordenadores e Internet, en diseño de base de datos, hojas de cálculo, uso de software colaborativo de trabajo y manuales de ayuda en línea, comprensión de compatibilidad de hardware y software, y en la mejora de producciones multimedia.

Con respecto al "Uso de aulas universitarias de informática", los datos señalan que los estudiantes que usan dichas aulas poseen un mejor conocimiento del funcionamiento de foros universitarios que aquellos que no las utilizan.

Algunos estudios previos muestran resultados similares respecto a las diferencias de género en el manejo de las TIC, y donde se muestra cómo los hombres poseen una visión y actitud más positiva que las mujeres con respecto a su uso (Espinar y González, 2009; Romero, 2011). No obstante, nuestros resultados señalan que estas distinciones entre género en la utilización de las TIC desaparecen en el dominio de conocimientos básicos y moderados de las herramientas digitales. Por ello, la diferencias entre género en el uso digital se limita al conocimiento complejo de las herramientas tecnológicas que van surgiendo en la sociedad.

En la actualidad, la Sociedad de la Información se caracteriza por la entrada continúa de nuevas herramientas en nuestra sociedad. Por ello, es 
necesario que las personas conozcan y manejen de forma adecuada y precisa estas herramientas, y así, poder formar parte de la construcción de nuestra sociedad. Cabe destacar el área educativa, donde cada vez más están presentes los laboratorios tecnológicos y enseñanzas virtuales (Blanco y Anta, 2016). De hecho, para poder formarse en enseñanza superior los estudiantes deben tener un buen conocimiento y poseer importantes habilidades en las enseñanzas virtuales.

Siguiendo la revisión científica realizada por Cabero et al. (2016), las herramientas tecnológicas en el ámbito educativo y especialmente en la enseñanza superior aportan las siguientes ventajas en la formación de los estudiantes:

Permiten la creación natural de una inteligencia colectiva, amplían las fronteras del proceso de enseñanza-aprendizaje, facilitan la comunicación, favorecen la construcción colaborativa del conocimiento, implican un cambio en la gestión de los procesos de enseñanza, fomentan una visión compartida del conocimiento, favorecen la socialización del grupo, son espacio de encuentro entre los diferentes actores del proceso enseñanza aprendizaje; permiten centralizar en un único sitio todas las actividades docentes, aumentan la fluidez y sencillez de la comunicación entre profesores y alumnos, facilitan la coordinación y trabajo de diversos grupos de aprendizaje, aumentan la interacción entre profesores y alumnos, facilitan la retroalimentación de los estudiantes a tiempo, mejora el aprendizaje. (p.3)

Aunque es verdad que hay una tendencia, por parte de las mujeres, en la incorporación en el uso de las TIC, podemos afirmar que todavía las herramientas tecnológicas están orientadas a los hombres (De la Garza y Tellez, 2016).

Algunos estudios señalan la diferencia en el uso de la tecnología entre hombres y mujeres como un problema social muy importante que es necesario erradicar en todos los ámbitos sociales $\mathrm{y}$, por supuesto, también en las aulas escolares de todos los niveles educativos. La presencia de mujeres en titulaciones tecnológicas como informática y telecomunicaciones sigue siendo muy baja. El Ministerio de Educación, Cultura y Deportes (2015) apuntaba que el número de matriculaciones de mujeres en carreras técnicas era muy escaso, alrededor del $26 \%$ del total de matrículas. De igual modo, muchos estudios realizados recientemente apuntan a la poca presencia femenina especializada en industrias tecnológicas (González-Palencia y Jiménez, 2016).

Según Barragán y Ruíz (2013), la transmisión de estas desigualdades representa una amenaza social importante, dándose una brecha digital que desfavorece a las mujeres y que, por tanto, es necesario que desaparezca. Son muchos los trabajos que señalan la necesidad de la incorporación de la mujer al uso de las TIC como una forma de eliminación de discriminación hacia la misma. De ahí, la importancia de incluir la igualdad de género en el manejo de las TIC (De la Garza y Tellez, 2016).

Por último, cabe preguntarse por qué el poco interés en el ámbito de la tecnología de las mujeres. Pues bien, estudios como los de González-Palencia y Jiménez (2016) apuntan a la socialización diferencial de género como causa del poco empeño de las mujeres en el aprendizaje del conocimiento y manejo de las TIC.

\section{CONCLUSIONES}

En conclusión, las mujeres presentan un escaso conocimiento y manejo de las herramientas tecnológicas en comparación con los hombres cuando comienzan su formación universitaria. Aunque en los conocimientos básicos y medios de las TIC no se observan diferencias de género, sí que existen diferencias entre hombres y mujeres en el manejo de las mismas cuando se requiere unos conocimientos más complejos. Posiblemente, el bajo interés de las mujeres en el ámbito tecnológico se deba a la socialización diferencial de género que provoca una diferenciación de roles entre hombres y mujeres.

\section{LIMITACIONES}

Este artículo presenta la tendencia del uso y percepción de las TIC y las diferencias de género en el manejo de estas herramientas digitales en alumnos que comienzan su formación universitaria en la titulación de Pedagogía de la Universidad de Málaga. Para futuras investigaciones, sería necesario aumentar la muestra e incluir en esta alumnos de otras titulaciones y universidades.

\section{AGRADECIMIENTOS}

Agradecemos a Juan Miguel Enamorado su excelente ayuda técnica en la realización del presente trabajo. 


\section{REFERENCIAS}

Aranda, L., Santacruz, M. I., Díaz-Ramos, A., López, M. M., Martín-Valero, R., y Ruiz-Párraga, G. (2015). Rúbrica: Evaluación de estrategias de aprendizaje aplicadas en diferentes grados y cursos académicos en la Universidad de Málaga. En Ruiz-Palmero, J., Sánchez-Rodríguez, J., y Sánchez-Rivas, E. (Edit.). Innovaciones con tecnologías emergentes (pp. 1-10). Málaga, España: Universidad de Málaga.

Barragán, R. y Ruíz, E. (2016). Brecha de género e inclusión digital. El potencial de las redes sociales en educación. Profesorado. Revista de currículum y formación del profesorado, 17(1), 309-323.

Blanco, A., y Anta, P. (2016). La perspectiva de estudiantes en línea sobre los entornos virtuales de aprendizaje en la educación superior. Innoeduca. International Journal of Techonology and Educational Innovation, 2(2), 109116.

Cabero, J., Barroso, J., Llorente-Cejudo, M. C., y Yanes, C. (2016). Redes sociales y Tecnologías de la Información y la Comunicación en Educación: aprendizaje colaborativo, diferencias de género, edad y preferencias. RED. Revista de Educación a Distancia, 1, 1-23.

Comas, M. A. (2013). El EEES, identidad y competitividad Europea: Principios fundamentales e interpretación de las principales autoridades. Revista de Docencia Universitaria, 11(1), 243-263.

De la Garza, R., y Tellez, E. (2016). Género y TIC. Por una sociedad de la información con perspectiva de género. En E. Tellez-Carvajal (Edit.), Derecho y TIC. Vertientes actuales (pp. 49-78). Mexico: Instituto de Investigaciones Jurídicas.

Díaz-Levicoy, D. (2013). TIC en Educación Superior: Ventajas y desventajas. Educación y Tecnología, 4, 44-50.

Espinar, E., y González, M. J. (2009). Jóvenes en las redes sociales virtuales: un análisis exploratorio de las diferencias de género. Feminismo/s, 14, 87-105.

Fernández-Cruz, F.J., Fernández-Dìaz, M.J. y RodríguezMantilla, J.M. (2018). El proceso de integración y uso pedagógico de las TIC en los centros educativos madrileños. Educación XX1, 21(2), 395-416.

García-Sánchez, M. R., Reyes-Añorve, J., y Godínez-Alarcón, G. (2017). Las Tic en la educación superior, innovaciones y retos. Revista Iberoamericana de las Ciencias Sociales y Humanísticas, 6(12), 1-18.

Gijón, J., y Crisol, E. (2012). La internalización de la Educación Superior. El caso del Espacio Europeo de Educación Superior. Revista de Docencia Universitaria, 10(1), 389414.

Giudicessi, S. L., Martínez-Ceron, M. C., Saavedra, S. L., Cascone, O., y Camperi, S. A. (2016). Las Tecnologías y la Enseñanza en la Educación Superior. Un Simulador Aplicado a la Integración de Conceptos Enseñados en Cursos de Posgrado. Revista Iberoamericana de Evaluación Educativa, 9(2), 9-28.

González-Palencia, R., y Jiménez, C. (2016). La brecha de género en la educación tecnológica. Ensaio: Avaliação e Políticas Públicas em Educação, 24(92), 743-771.

Grande, M., Cañon, R., y Cantón, I. (2016). Tecnologías de la información y la comunicación: evolución del concepto y características International Journal of Educational Research and Innovation (IJERI), 6, 218-230.

Gudín de la Lama, E., Lasala-Navarro, I. y Iturriaga-Barco, D. (2016).Didáctica de la competencia histórica en la sociedad digital. Revista de Educación, 375, 61-85.
Hernández-Sellés, N. (2015). El trabajo colaborativo en entornos virtuales en educación superior. (Tesis Doctoral Inédita). Universidad de A Coruña.

Honmy, R., y Vázquez, R. (2012). Formación del docente universitario en el uso de TIC: Caso Universidades Públicas y Privadas (U. de Carabobo y U. Metropolitana). Revista de Medios y Educación, 41, 163-171.

López-Navas, C. (2014). Educación superior y TIC: conceptos y tendencias de cambio. Historia y Comunicación Social, 19, 227-239.

Llorente-Cejudo, M. C., y Cabero, J. (2010). Desarrollo de un instrumento sobre Competencias TIC en alumnos universitarios. En Congreso Euro-ibeamericano de Alfabetización Mediática y Culturas Digitales Sevilla: Sevilla.

Ministerio de Educación, Cultura y Deportes (2015). Datos básicos del sistema universitario español. Curso 20132014. Madrid.

Morales, M., Ortíz, A. M., Trujillo, J. M., y Raso, F. (2015). Percepción del alumnado universitario acerca del uso e integración de las TIC en el proceso educativo de la Facultad de Educación de Granada. Innoeduca. International journal of technology and educational innovation, 1(2), 56-78.

Nakano, T., Garret, P., Vásquez, A., y Mija, A. (2014). La integración de las TIC en la educación superior: reflexiones y aprendizajes a partir de la experiencia PUCP. En Blanco \& Negro, 4(2), 65-76.

Pozo, C., y Bretones, B. (2015). Dificultades y retos en la implantación de los títulos de grado en las universidades españolas. Revista de Educación, 367, 147-172.

Rodríguez, J., Álvarez, V., Gil, J., y Romero, S. (2011). Cultura y Educación, 23(3), 323-340.

Romero, R. (2011). Género en el uso del e-learning en las universidades andaluzas. Profesorado. Revista de currículum y formación del profesorado, 15(1), 121-13.

Santacruz, I., Aranda-Garrido, M. L., y García-Moreno, A. (2013). Estrategias transversales de aprendizaje y uso de TICs. En Sánchez, J.; Ruiz, J. y Sánchez, E. (Coords.). Buenas prácticas con TIC en la investigación y la docencia (pp. 1-10). Málaga, España: Universidad de Málaga.

Santacruz, I., Díaz-Ramos, A., Aranda, L., Jiménez-Lara, A., Martín-Valero, R., López, M. M., Ruíz-Párraga, G., y Gutiérrez, A. (2015). Aplicación de estrategias de aprendizaje para la adquisición de competencias profesionales y su evaluación mediante una rúbrica integradora en diversos títulos de grado de la Universidad de Málaga. En Ruiz Rosillo, M. A. (Coord.). Educar para transformar: Aprendizaje Experiencial (pp 297-304. Madrid, España: Universidad Europea de Madrid.

Tobón, S., Pimienta, J., y García-Fraile, J.A. (2010). Secuencias didácticas: aprendizaje y evaluación de competencias. Naucalpan de Juárez, México: Pearson.

Vidal, C. (2012). El espacio Europeo de educación Superior y su implantación en las universidades españolas. Revista Catalana de dret públic, 44, 253-283. 